\title{
A review of HPLC technique covering its pharmaceutical, environmental, forensic, clinical and other applications
}

\author{
Gita Chawla $^{1^{*}}$, Krishna Kr. Chaudhary ${ }^{2}$ \\ ${ }^{1}$ Professor, ${ }^{1-2}$ Dept. of Pharmaceutical Chemistry, School of Pharmaceutical Education and Research, Jamia Hamdard(Deemed to be \\ University), Delhi, India
}

*Corresponding Author: Gita Chawla

Email: gchawla@jamiahamdard.ac.in

\begin{abstract}
HPLC is an analytical technique widely used for identification, separation, detection and quantification of various drugs and its related degradants. Appropriate mobile phase, stationary phase, column, column size, temperature, wavelength and gradient selection is important for the suitable compatibility and stability of drug as well as impurities and degradants. Most of the drugs as well as other compounds can be analyzed by HPLC method because of the several advantages like rapidity, specificity, accuracy, precision and ease of automation in this method. This article has been prepared with an aim to review different aspects of HPLC, such as principle, types, mode of separation, characteristics, instrumentation, important parameters and various applications of HPLC in different fields.
\end{abstract}

Keyword: HPLC, Analysis, Method development and Validation, Detection, Identification, Quantitation.

\section{Introduction}

High-performance liquid chromatography or High pressure liquid chromatography (HPLC) is a specific form of column chromatography generally used in biochemistry and analysis to separate, identify, and quantify the active compounds. ${ }^{1}$ HPLC is the method of choice for checking peak purity of new chemical entities, monitoring reaction changes in synthetic procedures or scale up, evaluating new formulations and carrying out quality control / assurance of the final drug products. ${ }^{2,3}$

HPLC is now one of the most powerful tools in analytical chemistry. It has the ability to separate, identify, and quantify the compounds that are present in any sample that can be dissolved in a liquid. HPLC is the most accurate analytical methods widely used for the quantitative as well as qualitative analysis of drug product and used for determining drug product stability. 4,5

High pressure liquid chromatography is the full form for HPLC and as given in the name, there is use of high pressure in the principle of its operation. Also due to its efficiency in analysis of compounds it is regarded as High performance liquid chromatography. Some have even gone to the extent of calling it as High patience liquid chromatography based on the long human time requirement and patience needed in its operation. HPLC is basically a highly improved form of column chromatography. Instead of allowing the solvent to drip through a column under just the force of gravity, it is externally forced through the column under high pressures of up to $400 \mathrm{~atm}$. This makes the chromatographic process a lot faster. It also allows the use of very small particle size for the column packing material which gives a much greater surface area for interactions between the stationary phase and the molecules flowing through it. Thus, it allows a much better separation of the components of the mixture. ${ }^{6,7}$ Now a days, highperformance liquid chromatography coupled with tandem mass spectrometry (HPLC-MS/MS) is one of the most versatile analytical tools available due to its rapid and diverse growth in applications in the analysis of drugs and metabolites from biological fluids.

In HPLC, the essential equipment consists of an eluent, reservoir, a high-pressure pump, and an injector for introducing the sample, a column containing the stationary phase, a detector and recorder. ${ }^{8}$ The development of highly efficient micro particulate bonded phases has increased the versatility of the technique and has greatly improved the analysis of multi component mixtures.

\section{Principle}

HPLC principle is that solution of sample is injected into a column of porous material (stationary phase) and liquid phase (mobile phase) is pumped at higher pressure through the column. The principle of separation followed is the adsorption of solute on stationary phase based on its affinity towards stationary phase. $^{2}$

HPLC is a special branch of column chromatography in which the mobile phase is forced through the column at high speed. As a result the analysis time is reduced by 1-2 orders of magnitude relative to classical column chromatography and the use of much smaller particles of the adsorbent or support becomes possible increasing the column efficiency substantially. ${ }^{10}$

\section{Types of HPLC \\ Normal Phase Chromatography}

In NP-HPLC the nature of stationary phase is polar and mobile phase is non-polar. In a mixture of components to be separated, those analytes which are relatively more polar will be retained by the polar stationary phase longer than those which are relatively less polar. Therefore the least polar component will elute first. The attractive forces which exist are mostly dipole-dipole and hydrogen bonding interaction.

It is first choice for mixtures of isomers and for preparative scale HPLC and second choice for lipophilic 
samples that cannot dissolve well in water-organic mixtures. ${ }^{11,12}$

\section{Reversed Phase Chromatography}

RP-HPLC has a non-polar stationary phase and polar or moderately polar mobile phase. RP-HPLC is based on the principle of hydrophobic interaction. In a mixture of components, those analytes which are relatively less polar will be retained by the non-polar stationary phase longer than those which are relatively more polar. Therefore the most polar component will elute first. ${ }^{11}$ Molecules that possess some degree of hydrophobic character can be separated by reversed phase chromatography with excellent recovery and resolution. ${ }^{13}$

\section{Size Exclusion Chromatography}

SEC, also called as gel permeation chromatography or gel filtration chromatography mainly separates particles on the basis of size. The column is filled with material having precisely controlled pore sizes, and the sample is simply screened or filtered. Larger molecules are rapidly washed through the column; smaller molecules penetrate inside the porous of the packing particles and elute later. This technique is widely used for the molecular weight determination of polysaccharides. ${ }^{14}$

\section{Ion Exchange Chromatography}

In Ion-exchange chromatography, retention is based on the attraction between solute ions and charged sites bound to the stationary phase. This technique is used almost exclusively with ionic or ionizable samples. The stronger the charge on the sample, the stronger it will be attracted to the ionic surface and thus, the longer it will take to elute. ${ }^{14,15}$

\section{Bio-Affinity Chromatography}

Separation based on specific reversible interaction of proteins with ligands. Ligands are covalently attached to solid support on a bio-affinity matrix, retains proteins with interaction to the column-bound ligands. ${ }^{16}$

\section{Mode of Separation ${ }^{17,18}$}

There are two modes of separation in HPLC, based on eluent composition.

\section{Isocratic}

Isocratic mode of separation includes constant eluent composition; means equilibrium conditions in the column and the actual velocity of compounds moving through the column are constant. The peak capacity is low and the longer the component is retained on the column the wider is the resultant peak.

\section{Gradient}

Gradient mode of separation includes varying eluent composition. This technique significantly increases the separation power of a system mainly due to increase of the apparent efficiency (decrease of the peak width). Peak width varies depending on the rate of the eluent composition variation.

\section{Characteristics of HPLC ${ }^{14,19,20}$}

HPLC as compared with the classical Liquid Chromatography (LC) technique is characterized by:

1. High resolution.

2. Small diameter $(4.6 \mathrm{~mm})$, stainless steel, glass or titanium columns.

3. Column packing with very small $(3,5$ and $10 \mu \mathrm{m})$ particles.

4. Relatively high inlet pressures and controlled flow of the mobile phase.

5. Continuous flow detectors capable of handling small flow rates and detecting very small amounts.

6. Rapid analysis.

HPLC is the method of choice for the analysis of:

1. Non Volatile Substances.

2. Substances with high polarity or ionic samples.

3. Substances with high molecular weight.

4. Thermally unstable and decomposable substances.

\section{Instrumentation $^{16,21,22}$}

The essential equipment consists of a high-pressure pump, and an injector for introducing the sample, a column containing the stationary phase, a detector and recorder. (Fig. 1)

\section{Injection of the Sample}

Septum injectors are available; using which sample solution is injected. An injector (sample manager or autosampler) is able to introduce [inject] the sample into the continuously flowing mobile phase stream that carries the sample into the HPLC column. A new advanced rotary valve and loop injector can be used to produce reproducible results. Typical sample volumes are 5-to 20-microliters $(\mu \mathrm{l})$.

\section{Pump}

A high-pressure pump (solvent delivery system or solvent manager) is used to generate and meter a specified flow rate of mobile phase, typically milliliters per minute. The pump suctions the mobile phase from solvent reservoir and forces it to column and then passes to detector. The operating pressure depends on column dimensions, particle size, flow rate and composition of mobile phase. Normal flow rates in HPLC are in the 1 to $2 \mathrm{ml} / \mathrm{min}$ range. Typical pumps can reach pressures in the range of 6000-9000 psi (400-to 600bar).

\section{Columns}

It is where the actual separation takes place. The column contains the chromatographic packing material needed to effect the separation. This packing material is called the stationary phase because it is held in place by the column hardware. It is stainless steel tube $5-25 \mathrm{~cm}$ in length and $2-$ $4.6 \mathrm{~cm}$ internal diameter. Material of packing is superficially porous or totally porous. 


\section{Detector}

The detector can determine (detect) the individual molecules that come out (elute) from the column. A detector serves to measure the amount of those molecules so that the chemist can quantitatively analyze the sample components. The detector provides an output to a recorder or computer that result in the liquid chromatogram (i.e., the graph of the detector response).

There are several ways of detecting when a substance has passed through the column. Generally UV spectroscopy is attached, which detect the specific compounds. Many organic compounds absorb UV light of various wavelengths. The amount of light absorbed will depend on the amount of a particular compound that is passing through the beam at the time.

\section{Interpreting the Output from the Detector}

The output is recorded as a series of peaks, each one representing a compound in the mixture passing through the detector and absorbing UV light. The area under the peak is proportional to the amount of substance, which is passed through detector, and this area can be calculated automatically by the computer linked to the display.

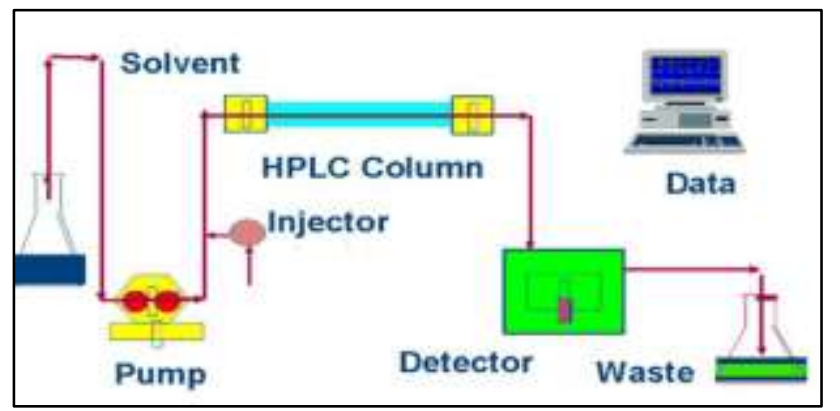

Fig. 1: Flow Diagram of HPLC ${ }^{2}$

\section{Some Important Parameters of HPLC ${ }^{23-25}$ System Resolution}

System resolution $=2(\mathrm{t} 2-\mathrm{t} 1) /(\mathrm{W} 2+\mathrm{W} 1)$ Where $\mathrm{t} 2$ and $\mathrm{t} 1$, are the retention times of the two components and $\mathrm{W} 2$ and $\mathrm{W} 1$ are the corresponding peak widths. The resolution factor should be greater than 2.00 for two HPLC peaks.

\section{Determination of System Precision}

After a standard solution is injected a number of times, the relative standard deviation of the peak responses is measured as either the peak height or peak area. When using an internal standard method, the response ratio is calculated. Maximum allowable system related standard deviations made at the $99 \%$ confidence level have been tabulated. For the USP monographs, five replicate chromatograms are used if the stated limit for relative standard deviation is $2 \%$ or less. Six replicate chromatograms are used if the stated relative standard deviation is more than $2.0 \%$.

\section{Asymmetry Factor or Tailing Factor}

The increase in the peak asymmetry is responsible for a decrease in chromatographic resolution, detection limits, and precision. Measurement of peaks on solvent tails should be avoided. The peak asymmetry factor or tailing factor can be calculated by following formula:

$\mathrm{T}=\mathrm{W} 0.05 / 2 \mathrm{f}$, Where W0.05 is the width of the peak at $5 \%$ peak height.

\section{Column Efficiency}

Column efficiency is generally determined by calculating the number of theoretical plates for a column. It is mainly required for the assay of antibiotics and antibiotic containing drugs.

$\mathrm{N}=5.545(\mathrm{t} / \mathrm{Wh} / 2)^{2}$ Or $\mathrm{N}=16(\mathrm{t} / \mathrm{W})^{2}$ Where $\mathrm{t}$ is the retention time of the analyte and $\mathrm{Wh} / 2$ is the peak width at half-height or $\mathrm{W}$ is the width at the base of the peak.

The height equivalent to one theoretical plate is calculated by $\mathrm{h}=\mathrm{L} / \mathrm{n}$ Where $\mathrm{L}$ is the length of the column.

\section{Column Capacity (Capacity factor / Retention factor)}

The column capacity factor is calculated by $\mathrm{K}=(\mathrm{tr}-\mathrm{tm}) /$ $\mathrm{tm}$, Where the retention time of the solute is $\mathrm{tr}$ and the retention time of solvent or un-retained substance is tm.

Retention volumes are sometimes preferred, because tr varies with flow rate. The factor is then calculated by $\mathrm{V}=$ $(\mathrm{Vr}-\mathrm{Vm}) / \mathrm{Vm}$, Where $\mathrm{Vr}$ is the retention volume of the solute and $\mathrm{Vm}$ is the elution volume of an unretained substance.

\section{Various Applications of HPLC Pharmaceutical Applications RP-HPLC method for simultaneous estimation of norfloxacin and tinidazole in tablet dosage form:}

An isocratic RP-HPLC method was developed for simultaneous separation and determination of norfloxacin and tinidazole in tablet dosage form and also in presence of some impurities within 2 minutes.

The method is rapid, accurate, selective, and reproducible. The main advantage of the method is the reduced analysis time due to monolithic silica columns. This analytical method is also adequate and useful for the estimation of norfloxacin and tinidazole in tablet for quality control laboratories, where low cost and fast analysis are essential. $^{26}$

\section{Simultaneous determination of thimerosal and} aluminum in vaccines and pharmaceuticals with the use of HPLC method:

A simple and convenient chromatographic method of simultaneous separation, identification, and quantitative determination of thimerosal (preservative) and aluminum (adjuvant) in vaccines and pharmaceuticals by RP-HPLC with visible (VIS) detection was developed and validated.

RP-HPLC method with the application of postcolumn derivatization with the use of dithizone as complexing agent makes it possible to determine Al and TM simultaneously. The presented method can be applied to vaccines, pharmaceuticals, and other products containing TM and Al. ${ }^{27}$ 
Development and validation of estimation of glimepiride and metformin by HPLC:

A simple and cost effective RP-HPLC method was developed for simultaneous estimation of glimepiride and metformin at tablet dosage form.

The results are highly specific and selective. The validated RP-HPLC method is simple, sensitive, precise and accurate for the simultaneous estimation studies in bioanalytical arena. ${ }^{28}$

Stability indicating RP-HPLC method for the determination of dronedarone hydrochloride and its potential process-related impurities in bulk drug and pharmaceutical dosage form:

A simple stability indicating analytical method for dronedarone was developed and validated using RP-HPLC technique. Developed method is used to evaluate the assay and related substances of dronedarone drug substance and tablets (Multaq ${ }^{\circledR}$ ). The drug substance was subjected to the stress conditions such as hydrolysis (acid and base), oxidation, photolysis and thermal degradation as per ICH prescribed stress conditions to show the stability-indicating the nature of the method.

The method is found to be simple, selective, precise, accurate and robust hence, this method can be used for routine testing as well as stability analysis of dronedarone hydrochloride drug substance and drug products. ${ }^{29}$

Development and validation of RP-HPLC method for glimepiride and its application for a novel selfnanoemulsifying powder (SNEP) formulation analysis and dissolution study:

A specific RP-HPLC method was developed for estimation of glimepiride and its dissolution study in selfnanoemulsifying powder (SNEP) formulation.

The proposed method is simple, precise, suitable, and accurate for quantification of glimepiride as an alternative to the existing methods for the routine analysis of glimepiride in pharmaceutical formulations and in vitro dissolution studies. $^{30}$

Development and validation of HPLC method for determination of human insulin in pharmaceutical preparation:

A simple and reliable HPLC method with diode array detection was developed and validated for determination of human insulin in pharmaceutical preparation. The method was validated for linearity, accuracy, precision, sensitivity and stability. Therefore, this method can be used for the routine QC analysis of human insulin in pharmaceutical preparations. $^{31}$

Determination of isotretinoin in pharmaceutical formulations by reversed-phase HPLC:

Isotretinoin finds its applications in the maintenance of epithelial tissues. An isocratic HPLC technique coupled with an UV-vis detector was developed for easy separation and quantification of 13-cis-retinoic (isotretinoin) from soft gelatin capsule formulations. The method was validated and the results showed good linearity, precision and accuracy for sensitive and selective quantitative determination of isotretinoin in the different pharmaceutical formulations. This method is not time-consuming and is easy to perform in any laboratory. ${ }^{32}$

Development and application of a validated HPLC method for the analysis of dissolution samples of levothyroxine sodium drug products:

Current USP methodology for levothyroxine (L-T4) is not adequate to resolve co-elutants from a variety of levothyroxine drug product formulations. So, a gradient HPLC method was developed for the analysis of dissolution samples of levothyroxine sodium tablets.

The method was validated according to USP Category I requirements. The method was found to be accurate, precise, selective, and linear for L-T4 over the analytical range. The HPLC method can be successfully applied to the analysis of dissolution samples of marketed levothyroxine sodium tablets. $^{33}$

Novel HPLC method for quantitative determination of cefazolin sodium in pharmaceutical formulations:

A validated HPLC method was developed for determination of cefazolin sodium in injectable pharmaceutical formulations. Forced degradation studies confirmed stability indicating power of this method.

This HPLC method is a simple, universal, convenient, and reproducible approach to identification and quantification of cefazolin sodium in injectable pharmaceutical formulations, and shows good performance. This method is simple, sensitive, and robust, requiring only a short chromatographic time to perform, and can be used for routine quality control in the laboratory and for therapeutic monitoring. ${ }^{34}$

A Stability indicating validated HPLC method for the determination of saxagliptin and metformin in bulk:

A simple HPLC method with UV detection was developed for the determination of saxagliptin and metformin in bulk.

The developed and validated HPLC method is selective, rapid and precise for the determination saxagliptin and metformin from bulk and it could be applied to the tablets. Since the method separates saxagliptin from its degradation products and tablet excipients it could also be used for quality control assay and for stability studies. The results obtained from the stress testing showed that saxagliptin is unstable under hydrolysis, oxidation and thermal stress. Therefore, care should be taken in the manufacturing process and during the storage of the product. ${ }^{35}$

Development and validation of HPLC method for quantification of norfloxacin in a pharmaceutical product:

A stability-indicating HPLC method was developed for the determination of norfloxacin in tablet dosage forms. For 
stress studies the drug was subjected to photolysis, oxidation, acid, alkaline and neutral conditions.

The proposed stability indicating HPLC method is found to be simple, sensitive, accurate, precise, linear, robust and specific for quantitative estimation of norfloxacin dosage forms. The analytical conditions and the solvent developed provided good resolution within a short analysis time and proved to be economical. The proposed method does not need sophisticated and expensive instrumentation. $^{36}$

Development and validation of Micro Emulsion High Performance Liquid Chromatography (MELC) method for the determination of nifedipine in pharmaceutical preparation:

There are two types of microemulsion which are used as a mobile phase; water in oil (w/o) and oil in water $(\mathrm{o} / \mathrm{w})$. Microemulsion has a strong ability to solubilize both hydrophobic and hydrophilic analytes, therefore reducing the pre-treatment of the sample which is needed for the complex sample. Separation of the analytes by using microemulsion HPLC can be achieved with superior speed and efficiency compared to conventional HPLC modes.

$\mathrm{O} / \mathrm{W}$ microemulsion mobile phase can be successfully applied and validated for the determination of nifedipine in aqueous solutions and in the pharmaceutical formulation. Moreover, it was rapid, precise and accurate. ${ }^{37}$

Micellar HPLC method for simultaneous determination of clonazepam and paroxetine $\mathrm{HCl}$ in pharmaceutical preparations using monolithic column:

A new and accurate micellar HPLC method coupled with ultraviolet detection was developed for simultaneous determination of Clonazepam and Paroxetine.

It could be applied to the analysis of both drugs in their single and laboratory prepared co-formulated dosage forms without any interference from the common excipients and the results show good agreement with those obtained by the comparison method. ${ }^{38}$

Validated method development for estimation of formoterol fumarate and mometasone furoate in metered dose inhalation form by HPLC:

A simple stability-indicating method was developed and validated for analysis of formoterol fumarate and mometasone furoate in metered dose inhalation formulations.

This is a simple, accurate, precise, and stabilityindicating HPLC method for the routine analysis of formoterol fumarate and mometasone furoate in metered dose inhalation formulations. The results of stress testing undertaken according to the ICH guidelines reveal that the method is selective and stability-indicating. ${ }^{39}$

Development and validation of RP-HPLC method for the determination of hydrochlorothiazide in bulk drug and pharmaceutical dosage form:
An HPLC-PDA method was developed and validated for the determination of hydrochlorothiazide in bulk and pharmaceutical formulation.

Successful application on pharmaceutical dosage tablet form gave high recovery of $99.93 \%$. The method was compared with official BP and other reported methods. The proposed method is economic, simple, and rapid and hence can be employed for routine analysis in quality control laboratories. $^{40}$

Simultaneous determination of 5 flavonoids and 7 saponins for quality control of Traditional Chinese Medicine preparation Xinnaoshutong capsule using HPLC-VWD ELSD:

Xinnaoshutong capsule (XC) is a traditional Chinese prescription derived from the ripe fruit of Tribulus terrestris L. (TT).

A reliable and simple method with HPLC-VWD-ELSD (High performance liquid chromatography-variable wavelength detector-evaporative light scattering detector) was developed and validated for simultaneous determination of twelve compounds in XC. The results of method validation illustrated that the presented method was precise, accurate, and sensitive as a practical technique for quantitative determination of multiple active components in XC. Twelve bioactive components including seven saponins and five flavonoids were selected as the chemical markers of XC to evaluate their quality of different batch samples.

Compared with traditional HPLC-UV method, HPLCVWD- ELSD could solve the problem of low UV absorption of saponins in order to achieve determination of multiple active components. The proposed method could be readily utilized as a routine analysis and effective tool to evaluate the quality control of Traditional Chinese medicines. $^{41}$

Development and validation of new RP-HPLC method for simultaneous determination of methyl and propyl parabens with levetiracetam in pure form and pharmaceutical formulation:

A simple and robust HPLC method was described for the assay for levetiracetam, methyl paraben, and propyl paraben either in their pure form or in commercial syrup.

The method was validated for linearity, accuracy, precision, specificity, limit of detection, limit of quantitation, robustness, and ruggedness. The complete separation of the analytes was achieved within short chromatographic run time (only $8 \mathrm{~min}$ ) with no interfering peaks allowing the analysis of a large number of samples in a short period of time. This gives the method high interest for routine sample analysis especially as no additional extraction or separation is required. ${ }^{42}$

A simple and improved HPLC-PDA method for simultaneous estimation of fexofenadine and pseudoephedrine in extended release tablets:

A simple RP-HPLC method was developed for simultaneous estimation of fexofenadine and 
pseudoephedrine in their extended release tablet. The method was validated according to the ICH and FDA guidelines and various validation parameters were determined. Also, forced degradation studies in acid, base, oxidation, and reduction media and in thermal condition were performed to establish specificity and stability indicating property of this method. This method can be applied in research laboratory and in pharmaceutical industries for routine analysis of fexofenadine and pseudoephedrine in their combined extended release tablets. $^{43}$

HPLC method for simultaneous determination of three curcuminoids in pharmaceutical dosage forms and other additives:

A reverse phase HPLC method was developed for the rapid determination of three curcuminoids viz. Curcumin (C), Desmethoxycurcumin (DMC) and Bisdesmethoxycurcumin (BDMC). The developed HPLC method is precise, specific, accurate and stability indicating for the determination of $\mathrm{C}$ $(95 \%)$. Statistical analysis also proves that the method is reproducible and specific for the analysis of $\mathrm{C}$ along with its co-purified derivatives DMC and BDMC. The method is versatile in the separate analysis of curcuminoids in bulk drug as well as in formulations such as, hydrogel nanoparticles, marketed $\mathrm{C}$ capsules and stabilizers, surfactants and co-surfactants. ${ }^{44}$

Determination of adsorption isotherms by means of HPLC for adsorption mechanism elucidation and separation optimization:

Adsorption isotherm can be determined by means of HPLC. To develop preparative chromatographic purification methods or to better understand the nature of the interactions between solute molecules and the surface of the stationary phase, adsorption isotherms have to be measured. In preparative chromatography, the information provided by the isotherms and by the proper model of chromatography will help the scale-up and optimization of batch and continuous purifications with minimum use of solvents and sample. There are great applications of these techniques for pharmaceutical applications, characterization of binding mechanisms, bio-affinity studies, molecular and chiral recognition processes. ${ }^{45}$

\section{HPLC and LC/MS analysis of pharmaceutical container} closure system leachables and extractables:

The term "leachables" refers to impurities in pharmaceutical products whose origin is the pharmaceutical container closure system in either direct or indirect contact with the formulation. Potential leachables identified through laboratory studies of pharmaceutical container closure system components are referred to as "extractables." Extractables and leachables are most often chemical additives to plastic and elastomeric container closure system components, or organic residues on metal and glass surfaces. HPLC and LC-MS are used for the detection, identification and quantitation of extractables and leachables. These methods have thus wide application in the contexts of both regulatory and analytical sciences. ${ }^{46}$

\section{Environmental Applications}

Solid Phase Micro Extraction-HPLC (SPME-HPLC) analysis of pesticides:

Pesticides are an important and diverse environmental and agricultural species. Their determination in formulations, in feed and food, and in complex environmental matrices (e.g., water, soil, sludge, sediments, etc.) often requires separation methods capable of high efficiency, unique selectivity, and high sensitivity. Because pesticides (organophosporus, organochlorine, carbamate, dithiocarbamate, etc.) are carcinogenic, they are problematic for humans in the course of the food chain. Residual analyses were performed to find out the concentration and type of pesticides and their metabolites left in food at the time of consumption. SPME in combination with HPLC has a wide acceptability as an analytical technique. This has great advantages over the classical sampling techniques, which are time consuming and require larger samples and solvents. SPME-HPLC method can be used for detecting polar carbamate pesticides in clean water samples. ${ }^{47}$

Solid phase microextraction- HPLC, as a novel tool for the analysis of toxic metal ions:

There is a great applications of solid phase microextraction (SPME)-HPLC in the analysis of toxic metal species as these are important contaminants and are carcinogenic. Their determination in formulations, in feed and food, and in complex environmental matrices (e.g., waste water and industrial effluents) is very crucial. SPME is used for extraction and online desorption of analytes with the mobile phase of HPLC and subsequent detection by UV, ICP-MS or ESI-MS as detectors. Different SPME-HPLC methods can be used for the analysis of metallic species of As, Cr, $\mathrm{Pb}, \mathrm{Hg}$ and $\mathrm{Se}^{48}$

Chromatographic methods for the determination of active substances and characterization of their impurities in pesticide formulations:

HPLC can be very useful analytical methods for determination of active substances and their impurities in pesticide formulations. Because counterfeit plant protection products may cause losses of crops and threaten public health, food trade and the environment, the quality control of plant protection products is an essential part to ensure their quality and effective usage. The identification and quantification of impurities is becoming a more and more significant part in the control of formulated plant protection products because impurities affect the quality, stability and safety of these products.

HPLC combined with other techniques such as mass spectrometry allow reliable determination of the highest number of active substances contained in plant protection products, and their impurities, in the shortest possible time, followed by practical implementation of these developed 
methods for routine control of the products currently on the market. $^{49}$

\section{Forensic Applications}

HPLC analysis of monofluoro-S-triazine dye during the dyeing process:

HPLC technique was applied in order to monitor the hydrolysis and dye-fiber bond-forming during the dyeing process. The results showed that using a proper execution of calibration curves of the active and hydrolyzed form of the dye and defined equations, HPLC technique enables determining the exact amount of both dye forms anytime during dyeing. Complete control of dyeing procedure is possible, since it is able to define the exact amount of the hydrolyzed and active form of the dye that was adsorbed and bonded with fibers according to calibration curves made for active and hydrolyzed form of the dye. ${ }^{50}$

Development of analytical methods to determine monitorable drugs in serum and urine by micellar liquid chromatography using direct injection:

Therapeutic drug monitoring is a common practice in clinical studies. It requires the quantification of drugs in biological fluids. Micellar liquid chromatography (MLC), a well-established branch of Reverse Phase-High Performance Liquid Chromatography (RP-HPLC), has been proven as a useful tool for the analysis of these matrices. MLC is used for determination of several groups of drugs in serum and urine such as anticonvulsants, antiarrhythmics, tricyclic antidepressants, selective serotonin reuptake inhibitors, analgesics and bronchodilators. The main advantage of this method is possibility of direct injection, and the efficient chromatographic elution, in spite of the complexity of the biological fluids. It was found that the MLC-procedures are fast, simple, inexpensive, ecofriendly, safe, selective, enough sensitive and reliable. Therefore, this is an excellent alternative for the determination of drugs in serum and urine for monitoring purposes. ${ }^{51}$

\section{HPLC methods for the analysis of explosives}

HPLC is a powerful analytical tool for the analysis of the explosives. Explosives are present in wide complex matrices at the training and testing sites. The detection of explosives in different environmental samples such as surface and subsurface soil and water, plant and animal tissues, etc., showed that contamination by explosives is widespread and can reach the water table and also accumulate in plants. The application of the preconcentration methods in combination with HPLC is very useful for their detection at very low concentration level in sub ppb range. HPLC-UV system offers many advantages over the other systems for the analysis of explosives. ${ }^{52}$

\section{HPLC methods to directly detect drug glucuronides in biological matrices}

Detection of a drug and its glucuronide metabolite(s) is of great importance in interpretive forensic and clinical toxicology. Until recently, glucuronides were determined by cleavage of the glucuronide with an enzyme (e.g., $\beta$ glucuronidase) to yield the parent compound, which was subsequently detected, or via derivatization to a more volatile or detectable analogue. Direct detection of the glucuronide conjugates using HPLC overcomes the critical limitations of approaches that involve enzymatic cleavage procedures and/or derivatization. Thus HPLC coupled with various detectors offers direct method to determine glucuronides of various potentially abused drugs in human biological matrices.

These methods will allow estimations of metabolite concentrations and identification of parent compounds long after the drug has cleared from systemic circulation. These methods have become invaluable tools to both the clinical and forensic toxicology community. ${ }^{53}$

\section{Food and Flavour Applications}

A $100 \%$ water mobile phase HPLC-PDA analysis of melamine and related analogues:

A RP-HPLC method was utilized for detecting melamine (MEL) and related analogues, cyanuric acid (CYA), ammeline (AML), and ammelide (AMD), using a 100\% water mobile phase. The method shows high stability, significant linearity and satisfactory sensitivity. An inexpensive and harmless method for the simultaneous detection of MEL, CYA, AML, and AMD was developed that may be further applied to the quantification in foods.

A water mobile phase method, is harmlessness to the environment and to humans and has a short run time and high sensitivity. For the quantification in various foods, the proposed HPLC method will be applicable enough by performing a suitable sample preparation technique. ${ }^{54}$

\section{Development and validation of a single HPLC method for analysis of purines in fish oil supplements:}

Individuals with gout are advised to avoid the habitual intake of purine-rich foods such as meats, seafood, purinerich vegetables, and animal protein. An efficient RP-HPLC method was developed and validated for the specific analysis of the naturally occurring purines guanine, purine, theobromine, and adenine. These purines are often found in fish oil and seafood. This analytical method reported quantifies all four purines in fish oil in about 20 minutes.

This is a simple, efficient, reproducible RP-HPLC method the analysis of naturally occurring purines in fish oils. ${ }^{55}$

HPLC determination of mono-, poly- and hydroxycarboxylic acids in foods and beverages as their 2-nitrophenylhydrazides:

Direct derivatization in conjunction with HPLC is utilized for the determination of both free and total carboxylic acids in foods and beverages. Mono-, poly- and hydroxycarboxylic acids in foods and beverages can be directly converted into their hydrazine derivatives through the reaction with 2-nitrophenylhydrazine hydrochloride which absorb visible radiation. The advantage of using visible detection is that the chromatograms are simpler and more selective. 
This HPLC analyses permit the isocratic separations of those carboxylic acids in the samples with good accuracy, precision and sensitivity. This method enables a remarkably long column life-time and therefore the method is particularly suitable for routine determinations of carboxylic acids in foods and beverages. ${ }^{56}$

\section{Recent developments in the HPLC separation of phenolic food compounds-}

Phenolic compounds, as an important class of natural products, are the main bioactive constituents of many dietary and medicinal plants. They have been reported to show extensive benefits to human health, including antioxidant, anti-inflammatory, and anticancer activities. In most cases, phenolic compounds are present in plants as a series of analogues with similar structures and physicochemical properties. Rapid, accurate, and sensitive analytical techniques for their analysis in different kinds of food samples are becoming increasingly important from a nutritional standpoint. HPLC is the most widely used separation technique for these purposes. New techniques like ultrahigh-pressure liquid chromatography (UHPLC), hydrophilic interaction liquid chromatography (HILIC), and multidimensional LC have found increasing application. These techniques can be employed for chemical analysis of phenolic compounds in food samples. ${ }^{57}$

\section{Clinical Applications}

Development of HPLC methods for the determination of vancomycin in human plasma, mouse serum and broncho alveolar lavage fluid:

Two HPLC methods utilizing a protein precipitation technique were developed to analyze vancomycin in human plasma, mouse serum and bronchoalveolar lavage (BAL) fluid.

These assays are simple, reproducible and accurate. These analytical techniques were successfully applied to analyze vancomycin concentrations in human plasma, mouse serum and BAL samples with minimal interference of the host matrix. ${ }^{58}$

Validated HPLC method for determination of caffeine level in human plasma using synthetic plasma:

A simple and reliable HPLC method was utilized for the determination of the caffeine level in human plasma. Synthetic plasma was used to construct calibration curves and quality control samples to avoid interference by caffeine commonly present in donor's human plasma.

The described method, combining the use of a simple plasma protein precipitation procedure and synthetic plasma, provides sensitive, accurate, and precise measurement of caffeine levels in human plasma in the therapeutic range. ${ }^{59}$

Validation of HPLC method for the determination of lansoprazole in pharmaceutical dosage forms and human plasma:
A chromatographic method for the quantitative determination of lansoprazole in pharmaceutical combinations and human plasma was developed.

The HPLC assay of lansoprazole has been shown to be of general applicability to commercially available products and spiked human plasma samples. The method is accurate, precise and specific. The described HPLC assay can be easily applied for the quantification of the degradation products. $^{60}$

RP-HPLC method for the simultaneous determination of lisinopril and NSAIDs in API, pharmaceutical formulations and human serum:

HPLC method was developed, validated and applied for the simultaneous determination of lisinopril and NSAIDs in bulk, pharmaceuticals formulations and human serum.

This HPLC method provides a simple, universal, convenient and reproducible approach for the simultaneous identification and quantification that can be used to determine lisinopril and NSAIDs. So the proposed method can be used for the drug analysis in routine quality control. In addition, this method has the potential application to clinical research of drug combination, multi-drug pharmacokinetics and interactions. ${ }^{61}$

Development and application of multidimensional HPLC mapping method for O-linked oligosaccharides: An HPLC mapping method was developed for detailed identification of $O$-glycans including neutral, sialylated, and sulfated oligosaccharides. Furthermore, using this method, it was able to quantitatively identify isomeric products from an in vitro reaction catalyzed by $\mathrm{N}$-acetylglucosamine- $6 \mathrm{O}$ sulfotransferases and obtain $O$-glycosylation profiles of serum $\operatorname{IgA}$ as a model glycoprotein. The HPLC map provides a glycomics tool for unambiguous identification and quantitative profiling of $O$-glycans expressed on a variety of proteins of physiological and pathological interest. $^{62}$

Validated method for the determination of gemifloxacin in bulk, pharmaceutical formulations and human serum by RP-HPLC:

An isocratic RP-HPLC method was used for the determination of gemifloxacin in bulk, dosage formulations and human serum. This method was further applied for in vitro interactions of gemifloxacin with essential and trace elements.

The proposed HPLC method is simple, isocratic, rapid, specific, accurate and precise for determination of gemifloxacin in bulk, pharmaceutical dosage formulation and human serum. Hence, it can be recommended for the routine quality control and evaluation of clinical data of gemifloxacin. ${ }^{63}$

A simple and specific method for estimation of lipoic acid in human plasma by HPLC:

A rapid HPLC method for determination of lipoic acid (LA) in human plasma was developed and validated. 
This HPLC method for the estimation of LA in human plasma is simple, sensitive, reproducible and precise. The assay is less laborious, economical and less time consuming than other reported methods previously. The validated HPLC-UV method may be applied to the measurement of LA levels in clinical practice. Further the LA concentrations can be confirmed by LC-MS quantification; thereby this method may be applied in future studies with shorter run time thereby large number of samples can be quantitated. ${ }^{64}$

\section{Development and utilization of reversed phase HPLC methods for a series of therapeutic agents:}

Multiple drugs can be used through this process and standard methods are presented for the pharmaceutics; cefuroxime, clindamycin, dexamethasone, dicloxacillin, doxycycline, metronidazole, oxymetazoline, paclitaxel, tobramycin, and vancomycin. Each of these drugs were analyzed using Reversed-Phase HPLC utilizing a hydrophobic column. This work shows the ability to analyze multiple drugs with a single simple, quick, cost effective system.

These methods have been determined to be efficient for the drug at hand, and where applicable gradient methods allow for separation and detection of multiple compounds if called for by the experiment. With a well-planned and executed methodology, HPLC can be a powerful tool for the detection of a wide range of antibiotics while requiring only a single apparatus. ${ }^{65}$

Development and validation of a HPLC method for determination of telmisartan in rabbit plasma and its application to a pharmacokinetic study:

A simple and sensitive HPLC method with fluorescence detection for quantitation of telmisartan in rabbit plasma was developed and validated. This validated method was used successfully for analysis of plasma samples from a pharmacokinetic study.

Compared to other methods, this is a simple, specific, sensitive and rapid method for estimation of telmisartan from rabbit plasma. The method provided excellent selectivity and linearity, and has been successfully applied to pharmacokinetic study. ${ }^{66}$

Development of a HPLC method for the determination of tedizolid in human plasma, human serum, saline and mouse plasma:

A simple HPLC method was developed and validated to analyze tedizolid in human plasma, human serum, saline and CD-1 mouse plasma.

This methodology was successfully used to replicate the results obtained using a LC-MS/MS detection method. While LC-MS/MS detection is more sensitive, this current HPLC method is more cost effective and has the advantage of less variability. ${ }^{67}$

HPLC determination of ciprofloxacin hydrochloride and ornidazole in human plasma:
A rapid chromatographic method was developed for simultaneous determination of ciprofloxacin hydrochloride and ornidazole in human plasma by reverse phase HPLC method.

The method was successfully applied for simultaneous determination of ciprofloxacin and ornidazole in human plasma without any interference from the additives and endogenous substances. It is a simple, selective and sensitive method requiring inexpensive reagents. Sensitivity of the method is suitable for handling various plasma levels of the drug. This method can be used for clinical and pharmacokinetic studies. ${ }^{68}$

HPLC method for determination of clobetasol in rat plasma and its application to skin penetration:

A simple and sensitive HPLC method was developed for quantification of clobetasol (CLB) in rat plasma.

This method was successfully applied to the study of penetration of clobetasol from different zinc salts in rat for the first time. The method is also proficient in determination of large number of biological samples. ${ }^{69}$

Optimation and validation of analytical method of cotrimoxazole in tablet and plasma in vitro by HPLC:

A simple and reproducible HPLC method was developed for simultaneous determination of sulfamethoxazole and trimethoprim, which are also known as cotrimoxazole, in tablet and human plasma in vitro.

The developed analytical method for cotrimoxazole quantification in tablet and plasma samples showed good specificity, sensitivity, linearity, precision, and accuracy over the entire range of clinically significant and therapeutically achievable plasma concentrations, thereby enabling its use in bioequivalence trials. ${ }^{70}$

Quantification of malondialdehyde by HPLC-FL application to various biological samples:

Malondialdehyde (MDA) is stabile product of lipid peroxidation (LPO), and therefore MDA is frequently used as a biomarker of LPO. To determine MDA level in various biological samples (human plasma, fish liver tissue and cells in culture), an HPLC method with fluorescent detection was used.

The HPLC-FL method is rapid, accurate and reliable to follow the extent of LPO in various biological samples, particularly in samples in which a low level of MDA is expected, such as cells in culture. Owing to the rapid analytical process and run time, it can be used for routine analysis of MDA in clinical laboratory. ${ }^{71}$

\section{Quantitative analysis of drugs in biological matrices by} HPLC hyphenated to fluorescence detection:

HPLC coupled with fluorescence detection is of great utility in bioanalytical applications. Compared with conventional UV absorbance detection used in HPLC, fluorescence detection can greatly enhance the sensitivity leading to limits of detection similar to those obtained with mass spectrometry, offering a sensitive, robust and relatively 
inexpensive instrumental method. Analysis of those pharmaceutical compounds in different biological matrices can be performed using this technique, which are either naturally fluorescent or derivatized with a fluorescent agent, or chiral compounds. ${ }^{72}$

Gradient reversed-phase HPLC for separation of ionogenic analytes:

Gradient RP-HPLC is advantageous for separation of ionogenic analytes. $\mathrm{pH}$ - gradient RP-HPLC method provide improvements in analyte separations and peak shapes, and pKa determination.

Combined $\mathrm{pH} /$ organic-modifier gradient mode of RPHPLC allows optimization of separation of ionogenic analytes, along with a method for determining their biorelevant physico-chemical parameters [e.g., hydrophobicity $(\log \mathrm{kw})$ and acidity $(\mathrm{pKa})]$. The method is applicable to drugs and other xenobiotic mixtures, including individual analytes of interest assayed in biological fluids. ${ }^{73}$

\section{Application of micro- and nano-HPLC to the determination and characterization of bioactive and biomarker peptides:}

Micro/nano-HPLC has a wide application to the analysis of bioactive peptides in biological matrices. The micro/nanoHPLC can be used in peptidomics to discover new endogenous bioactive peptides and to develop quantitation procedures to compare the levels of peptides of interest in two different biological samples. The application of micro/nano-HPLC with MS detection to the analysis of bioactive and biomarker peptides has demonstrated to be a better alternative to immunoassay methods. The high selectivity of micro/nano-HPLC-MS techniques solves the interference problems of cross reactivity associated to immunoassays.

The interest of the micro/nano-HPLC-MS techniques within the field of peptidomics is increasingly growing, in order to detect, identify, and quantify new endogenous bioactive and biomarker peptides in biological samples. ${ }^{74}$

\section{Miscellaneous Applications}

Application of HPLC method for the study of amino acid and peptide enantiomers:

Amino acids are very important organic compounds in nature. The biological activity of amino acids depends mainly on their stereoisomeric configuration (D- or L-). Thus, the stereochemical analysis of amino acids and peptides is an important aspect of their characterization. Among a wide range of analytical techniques available for the steroselective separation of different amino acids, which were obtained from plants or biological samples, HPLC like chromatographic are very useful.

The increasing development of the chiral systems used in steroisomeric analysis of amino acids enables direct separation of amino acid racemates using chromatographic techniques, but especially HPLC and GC. A modern trend in HPLC is two-dimensional chromatography. This innovation leads to decreased development time, increased amino acid resolution and detectability of amino acids in picomoles and femtomoles. $^{75}$

\section{Enantiomeric separation of nonproteinogenic amino acids by HPLC:}

Amino acids are essential for life, and have many functions in metabolism. One particularly important function is to serve as the building blocks of peptides and proteins. Peptides are frequently cyclic and contain proteinogenic as well as nonproteinogenic amino acids in many instances. Since most of the amino acids contain a chiral carbon atom, the stereoisomers of all these amino acids and the peptides in which they are to be found may possess differences in biological activity in living systems. The development of methods for the separation of enantiomers is of great interest, since the potential biological or pharmacological applications are mostly restricted to one of the enantiomers.

The separation of these isomers is achieved mainly by chromatographic and electrophoretic methods. Indirect and direct HPLC methods are useful for separation of biologically and pharmaceutically important enantiomers of nonproteinogenic amino acids and related compounds. ${ }^{76}$

\section{Recent developments in the HPLC separation of phenolic compounds:}

Phenolic compounds represent a class of highly complex naturally occurring molecules that possess a range of beneficial health properties. As a result, analysis of phenolics in a variety of samples is of great importance. HPLC is the basic method for phenolic separation. However, conventional HPLC methods provide insufficient resolving power when faced with the complexity of realworld phenolic fractions.

Nowadays there is an increasing demand for improved throughput and resolving power from the chromatographic methods used for phenolic analyses. A number of important technological advances in HPLC have demonstrated significant gains in terms of both speed and resolution. These include ultra-high pressure liquid chromatography (UHPLC), high-temperature liquid chromatography (HTLC), multi-dimensional separations as well as various new stationary phase chemistries and morphologies. These methodologies can contribute to improve performance in HPLC analysis of phenolics. ${ }^{77}$

Application of HPLC based measurements of lipophilicity to model biological distribution:

Octanol-water partition coefficients are the most widely used measure of lipophilicity in modelling biological partition/distribution. It has long been recognized that the retention of a compound in reversed-phase liquid chromatography is governed by its lipophilicity/hydrophobicity, and thus shows correlation with an octanol-water partition coefficient. HPLC provides a platform to measure various types of lipophilicity that can provide relevant information about the compounds' property. In this way HPLC can be more valuable than just an alternate for octanol-water partition. Chromatography 
using biomimetic stationary phases may provide better insight for biological partition/distribution processes. ${ }^{78}$

\section{Preparative HPLC based multidimensional chromatography and its application in Traditional Chinese Medicine:}

Separation of active components from Traditional Chinese medicine (TCM) is always difficult due to its complex matrix. The prep-HPLC-based multidimensional chromatography, which combined the characteristics of different separation techniques to improve separation capability and efficiency, is more conductive for separating and purifying complex TCMs.

Prep-HPLC-based multidimensional chromatography possesses a strong ability of separation and purification. It can improve the capacity and selectivity significantly and solve those segregation analysis problems that emerged in one dimension chromatographic separation mode. The development of multi-dimensional separation mode makes the automation of equipment improve significantly, as well as the reduction of production cycle and production cost. Prep HPLC-based multidimensional chromatography has become an important analysis tool in the study of TCMs gradually. ${ }^{79}$

\section{Conclusion}

HPLC is one of the most commonly used analytical technique. It is having several advantages over classical chromatographic techniques. Due to the simplicity and efficiency of HPLC, specific and rapid identification and determination of various natural and synthetic compounds can be carried out. It has wide applications in different fields such as pharmaceutical, environmental, forensic, food and flavour, clinical and many others in term of quantitative and qualitative estimation. The only disadvantage of HPLC is high cost.

Acknowledgements: Authors are thankful to Jamia Hamdard (Deemed to be University), New Delhi, India for the support.

\section{Conflict of Interest: None.}

\section{References}

1. Martin, M.; Guiochon, G. Effects of high pressure in liquid chromatography. J Chromatography A 2005;1090(1):16-38.

2. Dwivedia, S.K.; Agarwala, D.D. A Review: HPLC Method Development and Validation. Int J Analytical Bioanalytical Chem 2015;5(4):76-81.

3. Ahuja, S.; Rasmussen, H. Development for Pharmaceuticals, Separation Science and Technology, Elsevier: New York, 2007;8.

4. Rao, B.V.; Sowjanya, G.N.; Ajitha, A.; Rao, V.U. A review on stability indicating HPLC method development. World Journal of Pharmacy and Pharmaceutical Sciences. 2015;4(8):405-23.

5. Ahuja, S.; W dong, M. Handbook of Pharmaceutical analysis by HLPC, Elsevier: New York, 2005;6.

6. Kumar, V.; Bharadwaj, R. An Overview on HPLC Method Development. Optimization and Validation process for drug analysis, Pharm Chem J 2015;2(2);30-40.
7. HPLC-Chemiguide. www.chemguide.co.uk

8. Taleuzzaman, M.; Ahmed, M.M.; Chattopadhyay, M. Particle size role, Importance and Strategy of HPLC Analysis-An update. Int Arch BioMed Clin Res 2016;1(2):3.

9. Tamimi, L.; Dayyih, W.A.; Qinna, N.; Mallah, E.; Arafat, T. Pioglitazone $\mathrm{HCl}$ Levels and Its Pharmacokinetic Application in Presence of Sucralose in Animals Serum by HPLC Method. Pharm Analytica Acta 2014:5:318.

10. Sultana, N.; Arayne, S.; Shah, S.N. Development and validation for the simultaneous quantification of prazosin, amlodipine, diltiazem and verapamil in API, dosage formulation and human serum by RP-HPLC: application to in vitro interaction studies. Med Chem 2014;4:770-7.

11. Rao, G.; Goyal, A. An Overview on Analytical Method Development and Validation by Using HPLC. Pharm Chem J 2016;3(2):280-9.

12. Synder, L.R.; Kirkland, J.J.; Glajch, J.L. In: Practical HPLC Method Development, 2nd ed; John Wiley and Sons Inc: Canada, 1997.

13. Principles and Methods. In: Amesham Biosciences of Reversed Phase Chromatography. 1999;6-8.

14. Types of HPLC. http://hplc.chem.shu.edu/NEW/HPLC_Book/Introduction/int_t yps.html. (Accessed Feb 12, 2017)

15. Hearn, M.T. Ion-pair chromatography on normal and reversedphase systems. Advances in Chromatography. 1980;18:59-100.

16. Malviya, R.; Bansal, V.; Pal, O.P.; Sharma, P.K. High performance liquid chromatography: a short review. J Global Pharma Technol 2010;2(5):22-6.

17. Azim, MD. Sabir; Mitra, Moloy; Bhasin, Parminder S. HPLC Method Development and Validation: A Review. International research Journal of Pharmacy. 2013;4(4);39-43.

18. Schellinger, A.P.; Carr, P.W. Isocratic and gradient elution chromatography: a comparison in terms of speed, retention reproducibility and quantitation. $J$ Chromatography $A$ 2006;1109(2):253-66.

19. Jeffery, G.H.; Bassett, J.; Mendham, J.; Denney, R.C. Titrimetric analysis. Vogel's textbook of quantitative chemical analysis, 5th edition; Longman Scientific and Technical, Essex: UK, 1989;372-3.

20. Connors, A.K. A Text Book of Pharmaceutical Analysis, 3rd ed.; A Wiley Interscience publication: New York, 2005;373400.

21. McCown, S.M.; Southern, D.; Morrison, B.E. Solvent properties and their effects on gradient elution highperformance liquid chromatography: III. Experimental findings for water and acetonitrile. J Chromatography A 1986;352:493509.

22. How Does High Performance Liquid Chromatography Work? http://www.waters.com

23. John, A. Adomovics. Chromatographic Analysis of Pharmaceuticals. Marcel Dakker, Second Edition Revised and Expanded.1-17.

24. Dr Sarma, K.P.; Murthy, Y.L.N.; Sarma, S.; Ramaiah, A. Basic Skills Training Guide-HPLC method development and validation- an overview, 2013.

25. Sneha, Lakshmi. R.P. A Review on Chromatography with High Performance Liquid Chromatography (HPLC) and its Functions. Research and Reviews: J Pharml Analysis 2015;4(1).

26. Sebaiy, M.M.; El-Shanawany, A.A.; Adl, S.M.; Abdel-Aziz, L.M.; Hashem, H.A. Rapid RP-HPLC method for simultaneous estimation of Norfloxacin and Tinidazole in tablet dosage form. Asian J Pharm Anal. 2011;1:79-84.

27. Zaręba, M.; Sanecki, P.T.; Rawski, R. Simultaneous determination of thimerosal and aluminum in vaccines and pharmaceuticals with the use of HPLC method. Acta Chromatographica 2015:1-3. 
28. Kumar, S.S.; Nakasthra, P.; Mamatha, K.; Yamuna, P.; Swamy, J. Development and Validation of Glimepiride and Metformin in Human Plasma by HPLC: An application study. Int $J$ Adv Pharm Analysis 2015;5(3):51-7.

29. Landge, S.B.; Jadhav, S.A.; Nimbalkara, K.P.; Mali, A.C.; Mathad, V.T. Stability indicating RP-HPLC method for the determination of dronedarone hydrochloride and its potential process-related impurities in bulk drug and pharmaceutical dosage form. American Journal of Analytical Chemistry. 2013, 4(06), 323.

30. Mohd, A.B.; Sanka, K.; Gullapelly, R.; Diwan, P.V.; Shastri, N. Development and validation of RP-HPLC method for glimepiride and its application for a novel selfnanoemulsifying powder (SNEP) formulation analysis and dissolution study. J Anal Sci Technol 2014;5(1):1.

31. Yilmaz, B.; Kadioglu, Y. Development and validation of HPLC method for determination of human insulin in pharmaceutical preparation. Int J Pharm Sci Rev Res 2010;2(2).

32. Guimarães, C.A.; Menaa, F.; Menaa, B.; Lebrun, I.; QuencaGuillen, J.S.; Auada, A.V.et al, Determination of isotretinoin in pharmaceutical formulations by reversed-phase HPLC. Journal of Biomedical Science and Engineering, 2010;3(5):454

33. Collier, J.W.; Shah, R.B.; Bryant, A.R.; Habib, M.J.; Khan, M.A.; Faustino, P.J. Development and application of a validated HPLC method for the analysis of dissolution samples of levothyroxine sodium drug products. J Pharm Biomed Anal 2011;54(3):433-8.

34. Hasan, N.; Sher, N.; Siddiqui, F.A.; Ahmad, M.; Shafi, N.; Sial, A.A et al. Novel HPLC method for quantitative determination of cefazolin sodium in pharmaceutical formulations. Res Rep Med Chem 2013;3(3):21-8.

35. Caglar, S.; Alp, A.R. A validated high performance liquid chromatography method for the determination of saxagliptin and metformin in bulk, a stability indicating study. J Anal Bioanalytical Tech 2014;S12: 010.

36. Chierentin, L.; Salgado, H.R. Development and validation of a simple, rapid and stability-indicating high performance liquid chromatography method for quantification of norfloxacin in a pharmaceutical product. J Chromatography Separation Tech 2013;171-5

37. AL-Jammal, M.K.; Al Ayoub, Y.; Assi, K.H. Development and Validation of Micro Emulsion High Performance Liquid Chromatography (MELC) Method for the Determination of Nifedipine in Pharmaceutical Preparation. Pharm Anal Acta 2015;6(3).

38. Ibrahim, F.; El-Enany, N.; Shalan, S. Micellar High Performance Liquid Chromatographic Method for Simultaneous Determination of Clonazepam and Paroxetine $\mathrm{HCl}$ in Pharmaceutical Preparations Using Monolithic Column. J Chromatogr Sep Tech 2016;7:331.

39. Srinivasarao, K.; Gorule, V.; Ch, V.R. Validated method development for estimation of formoterol fumarate and mometasone furoate in metered dose inhalation form by high performance liquid chromatography. J Anal Bioanalytical Tech, 2012;3(6):301-6.

40. Mohammed, N.S.; Mohammed, A.J. Development and Validation of RP-HPLC Method for the Determination of Hydrochlorothiazide in Bulk Drug and Pharmaceutical Dosage Form. Chromatography Res Int 2016.

41. Li, J.; Bai, Y.; Zhang, P.; He, J.; Cao, J.; An, M.; Hu, L.M.; Gao, X.M.; Chang, Y.X. Simultaneous Determination of 5 Flavonoids and 7 Saponins for Quality Control of Traditional Chinese Medicine Preparation Xinnaoshutong Capsule Using HPLC-VWD-ELSD. J Anal Methods Chem 2017.

42. Abd El-Hay, S.S.; Mohram, M.S. Development and Validation of New RP-HPLC Method for Simultaneous Determination of
Methyl and Propyl Parabens with Levetiracetam in Pure Form and Pharmaceutical Formulation. Chromatography Res Int 2016.

43. Kayesh, R.; Sarker, A.S.; Sultan, M.Z.; Jahan, M.S. A Simple and Improved HPLC-PDA Method for Simultaneous Estimation of Fexofenadine and Pseudoephedrine in Extended Release Tablets by Response Surface Methodology. J Chem 2017.

44. Gugulothu, D.B.; Fernandes, C.B.; Patravale, V.B. A versatile high performance liquid chromatography method for simultaneous determination of three curcuminoids in pharmaceutical dosage forms. Pharm Anal Acta 2012.

45. Marchetti, N.; Cavazzini, A.; Pasti, L.; Dondi, F. Determination of adsorption isotherms by means of HPLC: Adsorption mechanism elucidation and separation optimization. J Separation Sci 2009;32(5-6):727-41.

46. Norwood, D.L.; Jenke, D.; Manolescu, C.; Pennino, S.; Grinberg, N. HPLC and LC/MS Analysis of pharmaceutical container closure system leachables and extractables. J Liquid Chromatography Relat Tech 2009;32(11-12):1768-827.

47. Aulakh, J.S.; Malik, A.K.; Kaur, V.; Schmitt-Kopplin, P. A Review on solid phase micro extraction - high performance liquid chromatography (SPME-HPLC) analysis of pesticides. Critical Rev Anal Chem 2005;35(1):71-85.

48. Malik, A.K.; Kaur, V.; Verma, N. A review on solid phase microextraction-High performance liquid chromatography as a novel tool for the analysis of toxic metal ions. Talanta 2006;68(3):842-9.

49. Płonka, M.; Walorczyk, S.; Miszczyk, M. Chromatographic methods for the determination of active substances and characterization of their impurities in pesticide formulations. TrAC Trends Anal Chem 2016;85:67-80.

50. Javorsek, D.; Kovac, F.; Gorensek, M. HPLC Analysis of Monofluoro-S-Triazine Dye during the Dyeing Process. Am J Anal Chem 2014;5(4).

51. Esteve-Romero, J.; Albiol-Chiva, J.; Peris-Vicente, J. A review on development of analytical methods to determine monitorable drugs in serum and urine by micellar liquid chromatography using direct injection. Anal Chimica Acta 2016;926:1-6.

52. Gaurav, D.; Malik, A.K.; Rai, P.K. High-performance liquid chromatographic methods for the analysis of explosives. Crit Rev Anal Chem 2007;37(4):227-68.

53. Kaushik, R.; Levine, B.; LaCourse, W.R. A brief review: HPLC methods to directly detect drug glucuronides in biological matrices (Part I). Anal Chimica Acta 2006;556(2):255-66.

54. Furusawa, N. A $100 \%$ Water Mobile Phase HPLC-PDA Analysis of Melamine and Related Analogues. Am J Anal Chem 2012;3(4):295.

55. Roy, L.; Harrell, C.C.; Ryan, A.S.; Thorsteinsson, T.; Sancilio, F.D. Development and Validation of a Single HPLC Method for Analysis of Purines in Fish Oil Supplements. Food Nutr Sci 2013;4(12): 1255

56. Miwa, H. High-performance liquid chromatographic determination of mono-, poly-and hydroxycarboxylic acids in foods and beverages as their 2-nitrophenylhydrazides. $J$ Chromatography A 2000;881(1):365-85.

57. Pyrzynska, K.; Sentkowska, A. Recent developments in the HPLC separation of phenolic food compounds. Crit Rev Anal Chem 2015;45(1):41-51.

58. Hagihara, M.; Sutherland, C.; Nicolau, D.P. Development of HPLC methods for the determination of vancomycin in human plasma, mouse serum and bronchoalveolar lavage fluid. $J$ Chromatogr Sci 2013;51(3):201-7.

59. Alvi, S.N.; Hammami, M.M. Validated HPLC method for determination of caffeine level in human plasma using 
synthetic plasma: application to bioavailability studies. $J$ Chromatographic Sci 2011;49(4):292-6.

60. Al-Momani, I.F.; Rababah, M.H. Validation of HPLC and FIA Spectrophotometric Methods for the Determination of Lansoprazole in Pharmaceutical Dosage Forms and Human Plasma. Am J Anall Chem 2010;1(01):34.

61. Sultana, N.; Arayne, M.S.; Siddiqui, R.; Naveed, S. RP-HPLC method for the simultaneous determination of lisinopril and NSAIDs in API, pharmaceutical formulations and human serum. Am J Anal Chem 2012;3(2):147.

62. Yagi, H.; Ohno, E.; Kondo, S.; Yoshida, A.; Kato, K. Development and application of multidimensional HPLC mapping method for O-linked oligosaccharides. Biomol 2011;1(1):48-62.

63. Sultana, N.; Arayne, M.S.; Shamim, S.; Akhtar, M.; Gul, S. Validated method for the determination of Gemifloxacin in bulk, pharmaceutical formulations and human serum by RPHPLC: in vitro applications. J Braz Chem Soc 2011;22(5):98792.

64. Ezhilarasi, K.; Sudha, V.; Ramachandran, G.; Umapathy, D.; Rajaram, R.; Padmalayam, I. et al; A Simple and Specific Method for Estimation of Lipoic Acid in Human Plasma by High Performance Liquid Chromatography. J Chromatography Separation Tech 2014;672(2):277-81.

65. Olbrich, J.; Corbett, J. Development and Utilization of Reversed Phase High Performance Liquid Chromatography Methods for a Series of Therapeutic Agents. Modern Chem Appl 2013;1:101.

66. Virkar, P.S.; Pingale, S.G.; Mangaonkar, K.V. Development and validation of a high performance liquid chromatography method for determination of telmisartan in rabbit plasma and its application to a pharmacokinetic study. J Anal Bioanalytical Techniques 2012;3:133.

67. Santini, D.A.; Sutherland, C.A.; Nicolau, D.P. Development of a High Performance Liquid Chromatography Method for the Determination of Tedizolid in Human Plasma, Human Serum, Saline and Mouse Plasma. J Chromatography Separation Tech 2015;6:270.

68. Rote, A.R. High Performance Liquid Chromatographic Determination of Ciprofloxacin Hydrochloride and Ornidazole in Human Plasma. Pharmaceutical Analytical Chemistry: Open Access 2015;1:103.

69. Musmade, P.B.; Deshpande, P.B.; Pathak, S.M.; Adiga, M.N.S.; Bhat, K.M.; Udupa, N. et al; High Performance Liquid Chromatographic Method for the Determination of Clobetasol in Rat Plasma and its Application to Skin Penetration. $J$ Bioanalysis Biomedicine 2010;2(1).
70. Mansur, U.; Sartika, J. Optimation and Validation of Analytical Method of Cotrimoxazole in Tablet and Plasma In vitro by High Performance Liquid Chromatography. $J$ Bioanalysis Biomedicine 2012;4:26-9.

71. Domijan, A.M.; Ralić, J.; Radić Brkanac, S.; Rumora, L.; Žanić-Grubišić, T. Quantification of malondialdehyde by HPLC-FL-application to various biological samples. Biomedical Chromatography 2015;29(1):41-6.

72. Lipka, E.; Vaccher, C. Quantitative analysis of drugs in biological matrices by HPLC hyphenated to fluorescence detection. Bioanalysis 2015;7(6):743-62.

73. Kaliszan, R.; Wiczling, P. Gradient reversed-phase highperformance chromatography of ionogenic analytes. TrAC Trends Anal Chem 2011;30(9):1372-81.

74. Saz, J.M.; Marina, M.L. Application of micro-and nano-HPLC to the determination and characterization of bioactive and biomarker peptides. J Separation Sci 2008;31(3):446-58.

75. Dołowy, M.; Pyka, A. Application of TLC, HPLC and GC methods to the study of amino acid and peptide enantiomers: a review. Biomedical Chromatography 2014;28(1):84-101.

76. Ilisz, I.; Aranyi, A.; Pataj, Z.; Péter, A. Enantiomeric separation of nonproteinogenic amino acids by highperformance liquid chromatography. J Chromatography A 2012;1269:94-121.

77. Kalili, K.M.; de Villiers, A. Recent developments in the HPLC separation of phenolic compounds. J Separation Sci 2011;34(8):854-76.

78. Valkó, K. Application of high-performance liquid chromatography based measurements of lipophilicity to model biological distribution. J Chromatography A 2004;1037(1):299-310.

79. Xiu-Man, X.I.; Wan-Yang, S.U.; HUANG JY, Polachi, N.; Ling, T.O.; Guo-Xiang, S.U. Preparative High Performance Liquid Chromatography-based Multidimensional Chromatography and Its Application in Traditional Chinese Medicine. Chinese J Anal Chem 2016;44(7):1140-7.

How to cite this article: Chawla G, Chaudhary KK, A review of HPLC technique covering its pharmaceutical, environmental, forensic, clinical and other applications. Int $J$ Pharm Chem Anal 2019;6(2):27-39. 